\title{
Performance Monitoring Framework for Service Oriented System Lifecycle
}

\author{
Tehreem Masood, Chantal Cherifi and Néjib Moalla \\ Decision and Information for Production Systems (DISP), Université Lumière Lyon 2, France \\ tehreem.masood@univ-lyon2.fr, chantal.bonnercherifi@univ-lyon2.fr,nejib.moalla@univ-lyon2.fr
}

\begin{abstract}
Keywords: Web Services, Service Oriented System, Quality of Service, Ontology, Technical Indicators and Performance.

Abstract: $\quad$ Service oriented systems are highly dynamic systems composed of several web services. One of the most important challenges in service oriented systems is to deliver acceptable quality of service. For this purpose, it is required to monitor quality of service along different activities of service oriented system. Existing research focuses on specific activities but do not take into account all the activities of service oriented system together at the infrastructure level. In this paper, we present performance monitoring framework to provide support for the whole service oriented system lifecycle. Our framework integrates several ontologies to monitor the performance of service oriented systems in order to ensure their sustainability. We design a base Service Monitoring Ontology that captures all the information about the service domain. Along with that we design ontologies for technical indicators at service level, binding level, composition level and server level. We conduct a performance evaluation over real web services using suitable estimators for response time, delay, loss and more.
\end{abstract}

\section{INTRODUCTION}

For many companies using big data path, collecting data has been a costly exercise with varying degrees of risk factors. Using big data is time consuming and hence lacks reactiveness which is not acceptable in real time decision making (Agrawal, 2015). These days understanding how real-time external data sources benefit an organization is vital.

Service oriented systems are highly dynamic systems composed of various web services providing by different service providers (Andrikopoulos, Bertoli and Bindelli, 2008). These systems are based on service oriented architecture (SOA) which provides interoperability, reusability, composition of web services and loose coupling (Erl, 2008). SOA allows realizing business processes by recursively combining services into orchestrations (Weerawarana et al, 2005). The business process is then itself exposed as a service to consumers. The standard language for service orchestration based on Web services is BPEL (BPEL 2.0 2007).

Service oriented architecture is used as a means to develop adaptive distributed software applications in a reactive manner. We can now develop techniques which take into account key data points to arrive at a more accurate real time predictions.

An important aspect in the service oriented system lifecycle is management of the performance along its lifecycle. Performance requirements on service or business processes are specified as technical indicators with target values which are to be achieved in a certain analysis period. Typical technical indicators are service duration, process duration, service response time, process response time and more. Due to the dynamic nature of service oriented systems, it is required to monitor Quality of Service (QoS) along various stages of its lifecycle. Technical indicators can be monitored at service or process execution time using $\mathrm{WSO} 2$, Oracle or Business Activity Monitoring technology.

Semantic web uses the notion of ontologies for the creation and elicitation of domain knowledge 
(Gomez-Perez et al, 2001). Ontologies represent formal specifications about the components of systems and their relationships in a machine understandable and processable manner (Antoniou et al, 2004). They play an important role in both semantic web applications and knowledge engineering systems (Fahad, Qadir, 2008). Several tasks such as information storage, processing, retrieval, decision making etc. are done on the basis of ontologies by such systems.

In this paper, we introduce a framework towards performance monitoring of service oriented system based on technical indicators. The framework consists of four major steps (i) Monitoring of performance to ensure its sustainability, (ii) Service monitoring ontology (iii) Ontologies for performance measurement of service oriented activities and (iv) Merging ontologies.

The remaining of the paper is organized as follows: Section 2 includes related work. Section 3 discusses performance measurement of service oriented activities. Section 4 discusses our proposed performance monitoring framework. We conclude the research in the last section.

\section{RELATED WORK}

In this section we discuss some techniques that have been proposed to ensure performance of web services.

\subsection{Performance Analysis of SOA Techniques}

In this section we analyze research that focuses on QoS.

(Tari et al 2011) proposed a benchmark of different SOAP bindings in wireless environments. Three sets of experiments were carried out: loopback mode, wireless network mode and mobile device mode. The experimental results show that HTTP binding inherits very high protocol overhead $(30 \%-$ $50 \%$ higher than UDP binding) from TCP due to the slow connection establishments and tear-down processes and the packet acknowledgement mechanism. UDP binding has the lower overhead because it does not require establishing connections before transmitting datagram's and does not address reliability. This results in a reduction in the response time and an increase in the total throughput. Its configuration and results can serve as a standard benchmark for other researchers who are also interested in the performance of SOAP bindings in wireless networks.

(Lin et al 2008) proposed an ontology based QoS-Aware support for semantic web services. They have used ontology to describe Quality of service metrics. They have composed their ontology into upper and lower level property. They have not considered the input/output operations. They have not included real time values for the performance analysis to reach towards optimal web service.

\subsection{Ontology based QoS Analysis Techniques}

(Moraes et al 2008) designed an ontology named MonONTO for proposing recommendation for the advanced internet applications users. They have considered information concerning the application type, traffic generated and user profile along with network performance metrics. Their expert system monitors the performance of advanced internet applications. Their ontology serves as a support to a decision reference tool by providing high-level information to the user about the agreement of the network facing the service level demands. They have used a fixed list of network parameters. Therefore, it does not deal with the heterogeneity and extensibility issues. Implementations of web services have not been done by them. Additionally, it does not deal with QoS mapping.

(Benaboud et al 2012) proposed Semantic Web Service Discovery Based on Agents and Ontologies considering the fuzzy constraints. Their framework is modelled by adding semantics of QoS attributes with web service profiles. It describes the design and implementation of a web service matchmaking agent. Agent uses an OWL-S based ontology and an OWL reasoner to compare ontology based service descriptions. They have used fuzzy constraints increases the efficiency of the web service discovery approach by providing the customers the web services which are not actually satisfying the input QoS constraints, but are close to the QoS constraints specification.

\subsection{QoS Aggregation and Performance Prediction Techniques}

QoS-based service selection has been done (Jaeger et al 2005) to describe how to find an optimal selection of functionally equivalent services based on their QoS properties. QoS aggregation as part of QoS-based service selection mostly addresses only QoS properties when interacting with other services (Unger 2005). It also neglects the time which is 
taken by the BPEL engine software to navigate through the process model (Rud 2007). The IT infrastructure consisting of hardware and software effects response time, throughput and availability of the service oriented system lifecycle. If the IT infrastructure is predefined, the goal is to estimate performance of service oriented system lifecycle (performance prediction) (Marzolla 2007), for example, by using benchmarking techniques.

\section{SERVICE ORIENTED ACTIVITIES FOR PERFORMANCE ANALYSIS}

An important aspect in the service oriented system (SOS) is management of the performance of different activities. There are different activities in service based systems to analyze performance like messaging, service, binding, business process and server. Service based activities for performance analysis are shown in Figure 1. Service is composed of different operations. Performance can be monitored at both the service and different operations level. Business process level is used for the performance analysis at the composition of service. Binding level covers the performance at the protocols level. Server level covers the performance of resources available. There are different types of technologies available which we can effectively utilize to guarantee better performance.

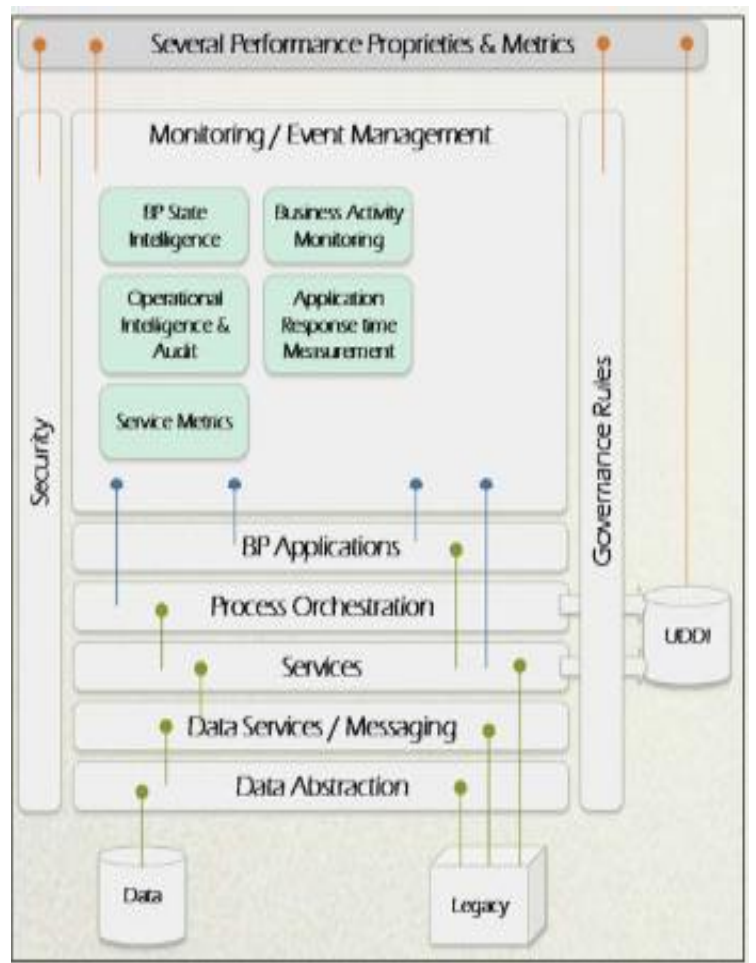

Figure 1: Service based Activities for Performance Analysis.

Data abstraction layer is used to query data from the database and the historical information about the data and components which is available in the legacy. The component services access the Data abstraction layer to fetch and retrieve data. Messaging through SOAP provides the ability to perform the necessary message transformation to connect the service requestor to the service provider and to publish and subscribe messages and events asynchronously (Tari et al 2011). In this way services are published in the service layer. Service oriented architecture is in process at the process orchestration layer. All this information is stored in the UDDI (Zhou et al 2009). On the top level layer, BP applications provide process orchestration mechanism to execute enterprise business processes. BP application use Business Process Modeling Notation (BPMN) to design their business processes. Governance rules are the set of policies like service will be available for one year etc. Security is used to provide some integrity to the system like authentication with the help of user name and password. Service metrics are the parameters in order to guarantee the performance of web services. For example in the ITIL(Donna 2014), there are 5 sub- categories and more than 100 metrics available for service support process and 5 sub- categories and 
more than 50 metrics available for service delivery processes. BP state intelligence is used to provide some intelligence or flags to measure the performance of BPEL.

\section{PERFORMANCE MONITORING FRAMEWORK}

This section presents our Performance Monitoring Framework. The first step of our approach is the specification of service or business process performance requirements. This specification is typically performed on user requirement or business goals in a certain time period. We divide our proposed performance monitoring framework into different steps as shown in Figure 2. We design performance based ontologies for all the activities of SOS. After the creation of these ontologies, we identify the common concept by merging these ontologies which results in performance profile.

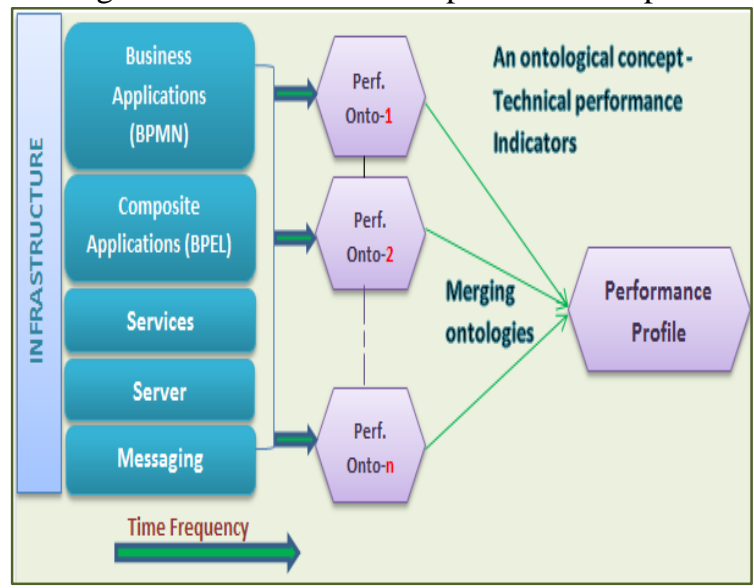

Figure 2: Creation of Performance Profile.

Performance requirements of SOS are monitored in terms of technical indicators. These technical indicators have target values that are required to achieve in a certain time period. For this purpose we perform performance analysis for SOS activities already explained in Section 3. We identify technical indicators at the service level, business process level, server level and binding level. We create ontologies for all these levels and merge these ontologies to create performance profile at the infrastructure level. Major steps for the creation of performance profile are:

- Specifying ontological concepts

- Management of common concepts

- Quality assurance on the performance profile
In the following sub sections we explain service monitoring ontology and ontologies at service level, server level, business process level and binding level.

\subsection{Service Monitoring Ontology (SMOnt)}

In this step we design a sophisticated Service Monitoring ontology (SMOnt) as a base infrastructure. It aggregates the main concepts and relationships between them. QoS requirements, service domain concepts, key performance indicators and performance levels are the major domains. SMOnt is shown in Figure 3.

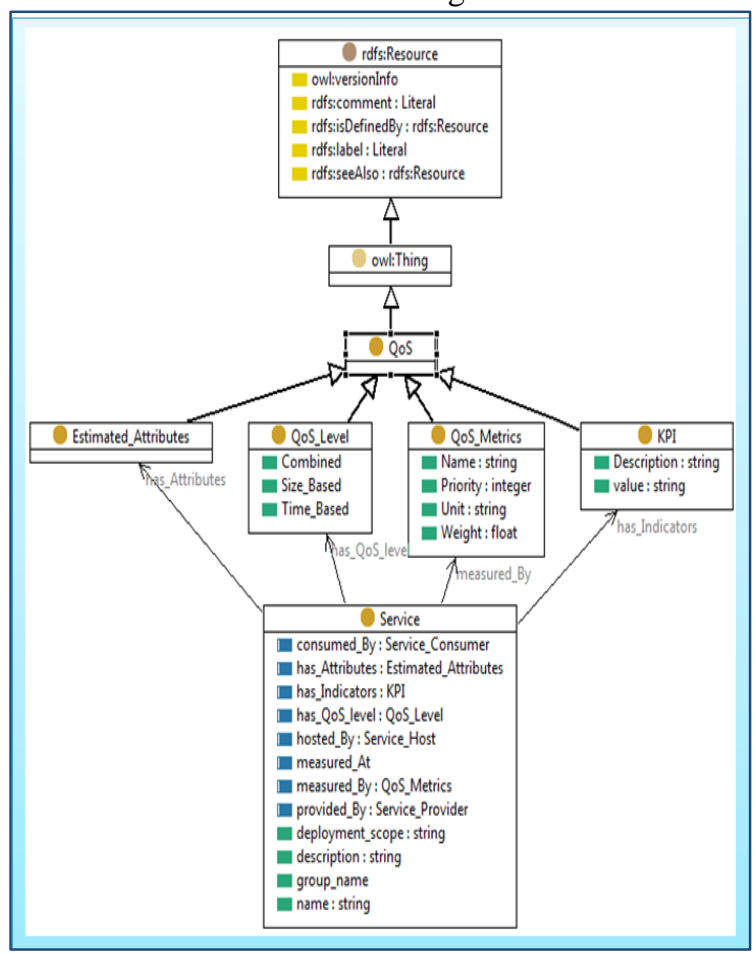

Figure 3: Service Monitoring Ontology (SMOnt).

Service: This concept has various data type properties to capture different attributes in SMOnt. It also has various object properties that links Service concept to other concepts. The details are as follows:

\section{Data type properties}

Name: records the name of the service.

Description: captures the description about the service.

Group_name: records name of the group to which the service belongs. 
Deployment_Scope: captures the deployment scope of the service.

\section{Object properties}

Consumed_By: captures the relationship of each service consumed by its consumers.

Has_Attributes: Each service has some attributes linked by this property between service and Estimated_Attributes

Has_Indicators: The performance of each service is monitored via various KPI and this property relates service with KPI concept.

Has_QoS_level: makes relation between service concept and QoS_Level.

Hosted_By: It captures the relation of each service hosted by its Host.

Measured_At: Each service is monitored and performance is measured at the Performance_Level.

Measured_By: Each service is measured by the QoS_Metrics via this property.

Provided_By: It captures the relation of each service provided by its provider.

Performance Level: This concept conceptualizes the level where a service network can be monitored. It has various sub concepts, each for capturing the performance levels such as Domain Level, Node level, Server Level, Service Level, Operation Level, and Messaging Level.

\section{QoS Level:}

Quality of service model is classified as metrics into time based, size based and combined (both time based and size based) metrics. Key performance indicator (KPI) assessment model has classified the indicators as response time, delay, error, loss, SLA, number of operations per second and average data blocks per time unit.

\section{Time based}

Time based classification includes all those indicators that can be measured in time units like availability, delay, response time. Availability is defined as the total down time per service. Delay is defined as downtime divided by uptime. Response time is also called latency. It is the time perceived by a client to obtain a reply for a request for a web service. It includes the transmission delays on the communication link. It is measured in time units.

\section{Size based}

Size based classification includes all those indicators that can be measured in size units. For example reliability. Reliability can be analyzed as loss or error of service. It is measured as number of successful invocations divided by total number of invocations.

\section{Combined}

Combined based classification includes all those indicators that can be measured by both time and size units like bandwidth and throughput. Bandwidth is defined as the tasks per time unit and average data blocks per time unit. Throughput is defined as the number of operations per second.

\subsection{Performance Ontologies}

In this step, we explain the ontological concepts of technical indicators at service level, business process level, binding level and server level. Ontologies for all these activities are shown below step by step. Figure 4 shows the ontology of technical indicators at service level.

Technical indicators at service level are explained below.

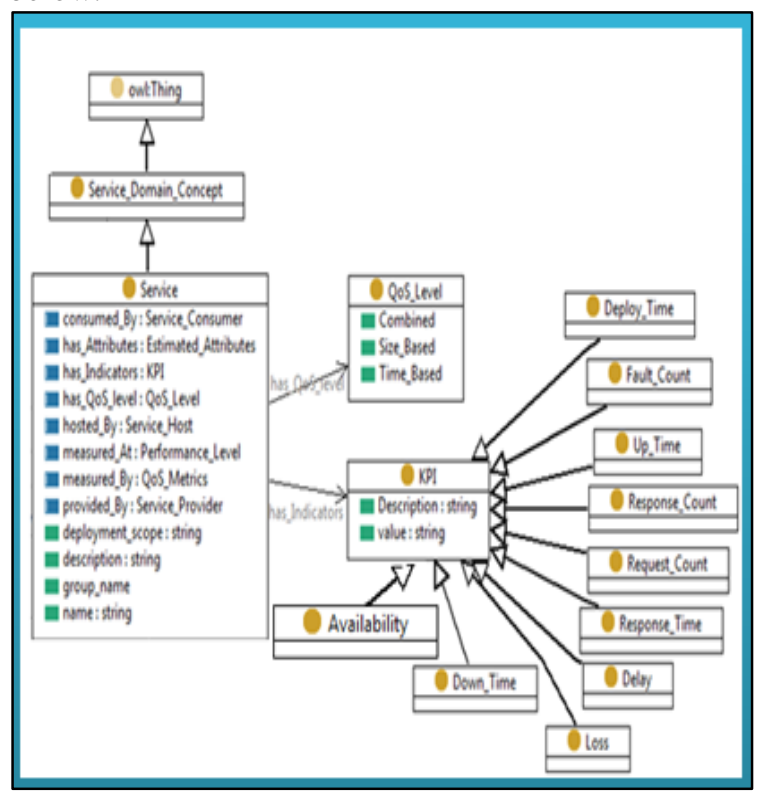

Figure 4: Ontology of Technical Indicators at Service Level.

Response Time: captures the response time of a service/operation. It has three sub concepts to record Maximum, Minimum and Average response time.

Request Count: shows the number of invocation of a service.

Response Count: shows the number of replies for an invocation of a service.

Fault Count: shows the number of invocations the service has not replied.

Deploy Time: shows when the service is deployed at the server

Up Time: shows the time period the service is available since its deployment 
Down Time: shows the time period of unavailability of a service since its deployment

Delay: shows the average response time of a service. Loss: shows that the service is un-available (i.e., it cannot be invoked).

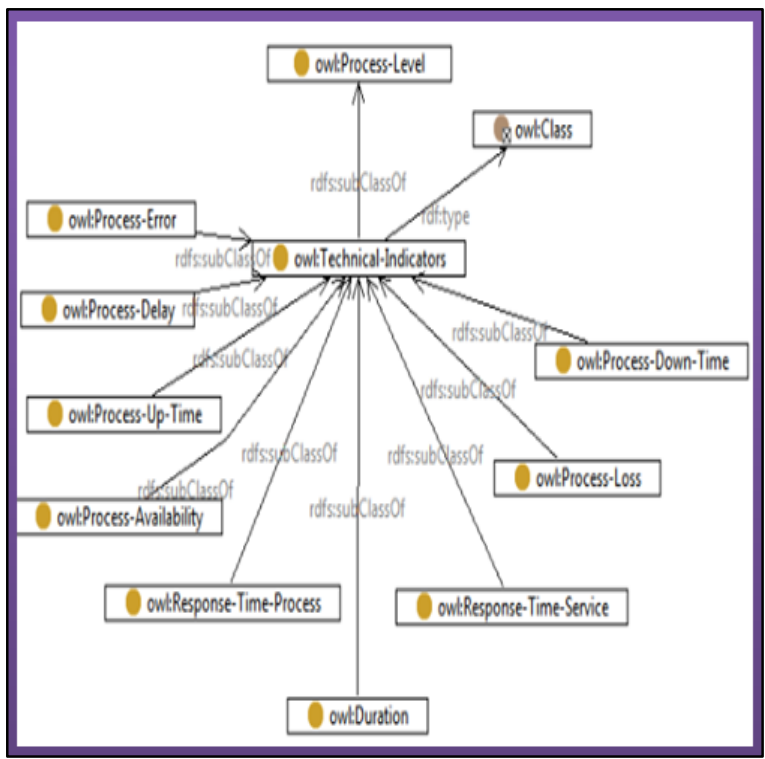

Figure 5: Ontology of Technical Indicators at Business Process Level.

Figure 5 shows the ontology of technical indicators at business process level. Technical indicators at business process level are explained below.

Process-Response-Time: captures the response time of a business process. It has three sub concepts to record Maximum, Minimum and Average response time.

Process-Up-Time: shows the time period the business process is available since its deployment Process-Down-Time: shows the time period of unavailability of a business process.

Process-Delay: shows the average response time of a business process.

Process-Loss: shows that the business process is unavailable (i.e., it cannot be invoked).

Process-Duration: shows the time duration of business process since it is deployed, executed and remained in process.

Some other technical indicators of service level are also used in order to estimate their value at composition level like availability and service response time.

Figure 6 shows the ontology of technical indicators at server level.

Technical indicators for the server level are free disk space, CPU Load, free RAM, throughput, bandwidth and reliability. We estimate available memory to support the new deployment by analyzing free disk space, CPU load and free RAM. Bandwidth is defined as the tasks per time unit and average data blocks per time unit. Throughput is defined as the number of operations per second.

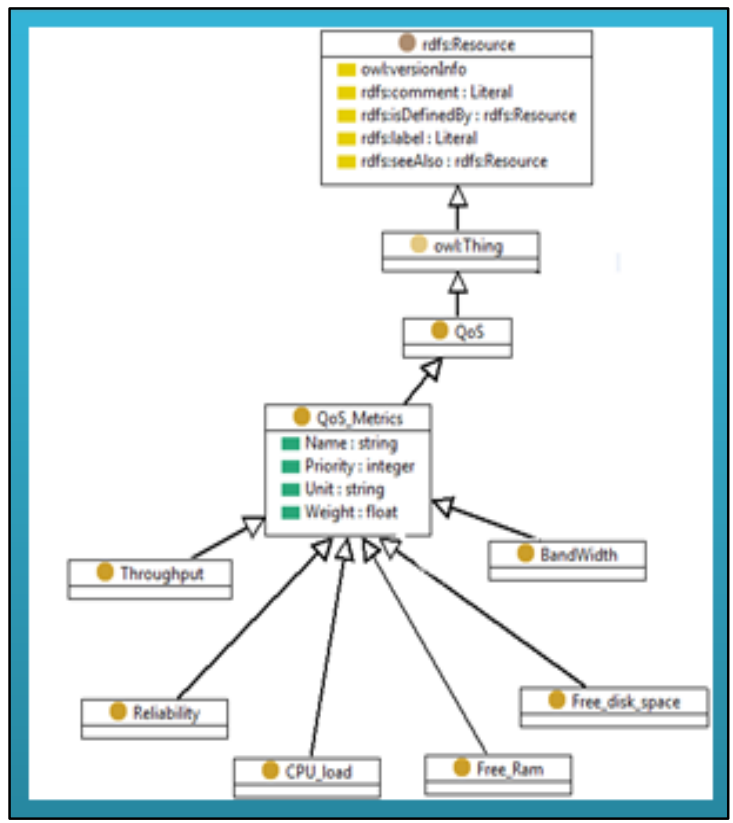

Figure 6: Ontology of Technical Indicators at Server Level.

Figure 7 shows the ontology of technical indicators at binding level. Binding level means at the messaging level. Technical indicators at transport messaging level are binding-throughput, bindingreliable-messaging, binding-security (authentication, authorization, and encryption), binding-bandwidth.

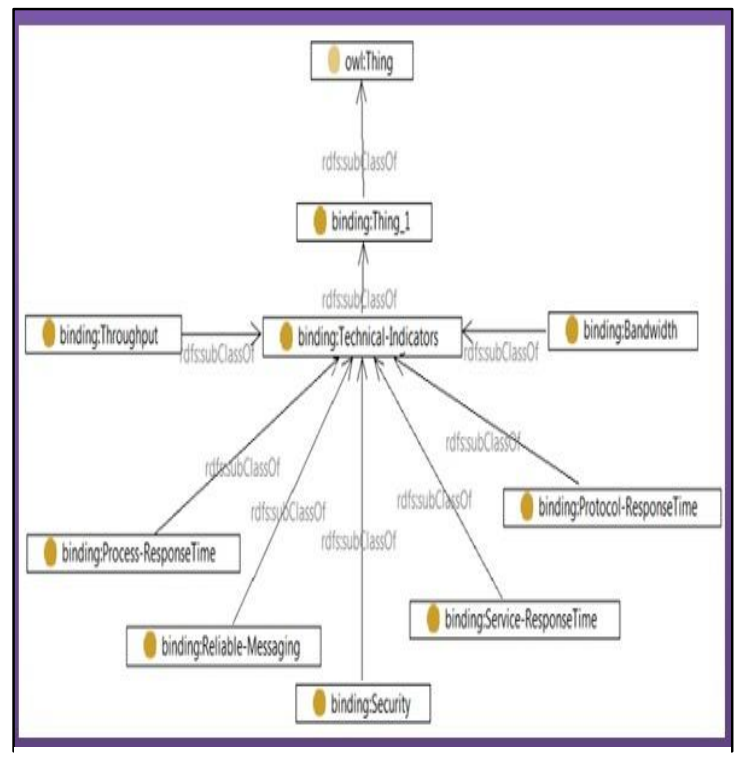

Figure 7: Ontology of Technical Indicators at Binding Level. 


\subsection{Merging Ontologies}

After designing ontologies for technical indicators of SOS activities, we merge them to create performance profile at the infrastructure level. There are three levels of analysis required for performance monitoring of SOS lifecycle.

\section{Level 1:}

In level 1, we try to increase the coverage of monitoring performance model. Also identification of sources of performance loss.

Level 2: Recommendations reengineering

Recommendations reengineering includes following steps.

- Traceability of the collected events

- Event Classification Model

- Operations for the re-engineering of components

- Number of instances of business application

At the business level:

- Assigning a job task to an available resource role.

At system level:

- Evolution of the specification of a service for better interoperability or better adaptation to specifications the overall architecture.

At the technical level:

- Need more hardware resources.

Level 3: User Demand Assessment

Recommendations reengineering includes following steps.

- Type of application

- Deploy a new component (i.e. service)

- Use an existing component

- Orchestrating existing components

- Assessment criteria:

- Compliance with the business logic

- Scope of the functional impact and dependency analysis

- Use of hardware resources

- Estimation of mounting costs and usage

We conduct a performance evaluation over real web services using suitable estimators for all the technical indicators at service level, business process level, server level and binding level. We perform this performance evaluation using WSO2 server and Oracle ${ }^{\circledR}$ Content Services Administrator.

\section{CONCLUSIONS}

In this paper we propose a performance monitoring framework to ensure sustainability of SOS at the infrastructure level. The framework consists of monitoring of performance, Service monitoring ontology, ontologies for performance measurement of service oriented activities and merging of ontologies. Extensive analysis of technical indicators with timing constraint to create performance profile is in process. We will implement our work by using real time case study.

\section{REFERENCES}

Agrawal, D, (2015) Challenges and Opportunities with. Big Data. White paper, USA.

Andrikopoulos, V., Bertoli, P., \& Bindelli, S. (2008).State. of the art report on software engineering design knowledge and survey of HCI and contextual knowledge. Project Deliverable PO-JRA.

Erl,T. (2008). Soa: Principles of service design(Vol.1).Prentice Hall Upper Saddle River.

Weerawarana, S., Curbera, F., Leymann, F., Storey, T., Ferguson, D. (2005) Web Services Platform Architecture. Prentice Hall PTR.

Web Services Business Process Execution Language Version 2.0 (2007) - Committee Specification. Published via Internet.

Antoniou, G., and Harmelen, F.V. 2004. A Semantic Web Primer. MIT Press Cambridge, ISBN 0-262-01210-3.

Fahad M. and Qadir, M. A. (2008). "A Framework for Ontology Evaluation.” ICCS Suppl, vol. 354, pp 149158.

Gomez-Perez, A., Lopez, M.F, and Garcia, O.C. (2001). Ontological Engineering: With Examples from the Areas of Knowledge Management, E-Commerce and the Semantic Web. Springer ISBN: 1-85253-55j-3.

Zhou, C., Chia, L. T., and Lee, B. S. (2009) "QoS-Aware Web Services Discovery with Federated Support for UDDI," Modern Technologies in Web Services Research, IGI Publishing Hershey New York.

Tari,Z., A. Phan, A. K., Jayasinghe,M., Abhaya, V. G. (2011) "Benchmarking Soap Binding. On the Performance of Web Services," pp 35-58, Springer.

Donna, K. (2014) A Guide to Service Desk Concepts: Service Desk and the IT Infrastructure Library, 4th edition, Course Technology Press Boston, MA, United States, ISBN- 10: 1285063457 ISBN-13: 9781285063454

Tari. Z, Phan. A. K. A, Jayasinghe. M, Abhaya. V. G,"Benchmarking. (2011) Soap Binding. On the Performance of Web Services," pp 35-58, Springer.

Lin L, Kai S, and Sen S. (2008) "Ontology-based QoSaware support for semantic web services," Technical Report at Beijing University of Posts and Telecommunications.

Moraes, P, Sampaio, L, Monteiro, J, Portnoi M. (2008): Mononto: A domain ontology for network monitoring and recommendation for advanced internet applications users. In: Network Operations and Management Symposium Workshops, IEEE.

Benaboud R, Maamri R, and Sahnoun Z. (2012)Semantic Web Service Discovery Basedon Agents and 
Ontologies, International Journal of Innovation, Management and Technology, Vol. 3, No. 4, pp 467472.

Jaeger, M.C., Muehl, G.; Golze, S. (2005) QoS-Aware Composition of Web Services: An Evaluation of Selection Algorithms. OTM Conferences.

Unger, T. (2005) Aggregation on QoS und SLAs in BPEL Geschaeftsprozessen Diplomarbeit Nr. 2305, Universitaet Stuttgart.

Rud, D, Kunz, M, Schmietendorf, A. (2007) Dumke, R.: Performance Analysis in WS-BPEL- Based Infrastructures. In Proc. "23rd Annual UK Performance Engineering Workshop" (UKPEW 2007), pp. 130-141.

Marzolla, M, Mirandola, R. (2007) Performance Prediction of Web Service Workflows, Proc. QoSA'07, Software Architectures, Components and Applications Third International Conference on Quality of Software Architectures, QoS A 2007, Medford, MA, USA, July 11-13. 